\title{
BMJ Open Multifactorial intervention to prevent cardiovascular disease in patients with early rheumatoid arthritis: protocol for a multicentre randomised controlled trial
}

Annemarie Lyng Svensson, ${ }^{1}$ Robin Christensen, ${ }^{2}$ Frederik Persson, ${ }^{3}$ Brian Bridal Løgstrup, ${ }^{4}$ Annamaria Giraldi, ${ }^{5}$ Christian Graugaard, ${ }^{6}$ Ulrich Fredberg, ${ }^{7}$ Jesper Blegvad, ${ }^{7}$ Tina Thygesen, ${ }^{7}$ Inger Marie Jensen Hansen, ${ }^{8}$ Ada Colic, ${ }^{9}$ Döne Bagdat, ${ }^{9}$ Palle Ahlquist, ${ }^{10}$ Hanne Slott Jensen, ${ }^{1}$ Kim Hørslev-Petersen, ${ }^{11}$ Ekta Sheetal, ${ }^{12}$ Torben Grube Christensen, ${ }^{13}$ Lone Svendsen, ${ }^{14}$ Henrik Emmertsen, ${ }^{15}$ Torkell Ellingsen ${ }^{12,16}$

\section{ABSTRACT}

Introduction: Cardiovascular morbidity is a major burden in patients with rheumatoid arthritis (RA). In this study, we compare the effect of a targeted, intensified, multifactorial intervention with that of conventional treatment of modifiable risk factors for cardiovascular disease (CVD) in patients with early RA fulfilling the 2010 American College of Rheumatology European League Against Rheumatism (ACR/EULAR) criteria. Methods and analysis: The study is a prospective, randomised, open label trial with blinded end point assessment and balanced randomisation (1:1) conducted in 10 outpatient clinics in Denmark. The primary end point after 5 years of follow-up is a composite of death from cardiovascular causes, nonfatal myocardial infarction, non-fatal stroke and cardiac revascularisation. Secondary outcomes are: the proportion of patients achieving low-density lipoprotein cholesterol $<2.5 \mathrm{mmol} / \mathrm{L}$, glycated haemoglobin $<48 \mathrm{mmol} / \mathrm{mol}$, blood pressure $<140 / 90 \mathrm{~mm} \mathrm{Hg}$ for patients without diabetes and $<130 / 80 \mathrm{~mm} \mathrm{Hg}$ for patients with diabetes and normoalbuminuria (urinary albumin creatinine ratio $<30 \mathrm{mg} / \mathrm{g}$ ) after 1 year of follow-up and the proportion of patients in each treatment group achieving low RA disease activity after 1 year, defined as a disease activity score C-reactive protein (DAS28-CRP) $<3.2$ and a DAS28-CRP score $<2.6$ after 12, 24 and 60 months. Furthermore, all hospitalisations for acute and elective reasons will be adjudicated by the event committee after 12, 24 and 60 months. Three hundred treatment-naive patients with early RA will be randomly assigned (1:1) to receive either conventional treatment administered and monitored by their general practitioner according to national guidelines (control group) or a stepwise implementation administered and monitored in a quarterly rheumatological nurse-administered set-up of behaviour modification and pharmacological therapy targeting (1) hyperlipidaemia, (2) hypertension, (3) hyperglycaemia and (4) microalbuminuria (intervention group).
Ethics and dissemination: This protocol is approved by the local ethics committee (DK-S2014007) and The Danish Health and Medicines Authority. Dissemination will occur through presentations at National and International conferences and publications in international peer-reviewed journals.

Trial registration number: NCT02246257

\section{INTRODUCTION}

Cardiovascular disease (CVD) is a major burden in patients with rheumatoid arthritis (RA), and the risk of myocardial infarction (MI) in patients with RA generally corresponds to the risk in 10-year older patients without RA. ${ }^{1}{ }^{2}$ Moreover, patients with RA have a reduced life expectancy. ${ }^{3}{ }^{4}$ In a meta-analysis that included 41490 patients with RA, the risk of CVD was increased by $48 \%$ and that of MI by $68 \%$ compared with the general population. ${ }^{5}$ The risk of CVD associated with RA is significantly increased compared with healthy individuals and is comparable with that seen in type 2 diabetes mellitus (T2DM). ${ }^{6}$ RA itself should be regarded as a strong independent cardiovascular risk factor for which cardiovascular risk management like that used in (T2DM) should be considered. ${ }^{6}$ It has been shown that the risk of both MI and stroke is doubled among patients with RA who visited their doctor at least thrice for RA during a 5-year period, compared with patients without RA visiting their doctor thrice. ${ }^{7}$ Furthermore, the risk of MI has been shown to be increased in young women within the first 3 years of RA treatment. ${ }^{28}$ 
Results from the Norfolk arthritis register showed that the risk of hospitalisation for CVD was approximately doubled in patients with early seropositive inflammatory RA compared with the general population. ${ }^{9}$ In a previous study, patients with RA had a substantially increased risk of death following MI but not stroke at 30-day follow-up, compared with patients without RA. ${ }^{10} 11$

Data from the Nurses' Health Study showed that patients with RA and hyperlipidaemia had an excessive risk of MI which was four times higher compared with patients with RA without hyperlipidaemia. ${ }^{12}$ More than $50 \%$ of patients with early RA had abnormal lipid levels at the time of diagnosis; however, increased inflammatory control for 2 years did not influence the hyperlipidaemic status. ${ }^{13}$ Discontinuation of statin therapy for $\geq 3$ consecutive months in patients with RA was associated with a $60 \%$ increased risk of death from CVD. $^{3}$

Patients with RA have an increased risk of sexual impediments, presumably due to a combination of biological, psychological, social and iatrogenic factors. ${ }^{14-17}$ Moreover, hyperlipidaemia is a well-known risk factor in the pathogenesis of sexual dysfunction. ${ }^{16-18}$ Endothelial dysfunction contributes to the pathogenesis of both CVD and common forms of erectile dysfunction (ED) and CVD. Some studies have shown that ED onset and severity are associated with an increased expression of markers of inflammation. ${ }^{18-20}$ The sexual ramifications of RA in combination with cardiovascular disease have, however, never been thoroughly investigated, and this cohort therefore offers a unique and prospective insight into the sexual function and quality of life of these patients.

To the best of our knowledge, this is the first study to investigate intensive interventions in a randomised setting aiming at prevention of CVD in treatment-naive patients with early RA.

\section{Rationale}

A targeted, intensified multifactorial intervention of modifiable risk factors for CVD in patients with T2DM with microalbuminuria is more effective in preventing cardiovascular death than conventional treatment. ${ }^{20} 21$ Thus, the primary aim of our present study is to evaluate the effect of a targeted, intensified, multidimensional intervention compared to conventional treatment of modifiable risk factors for CVD in patients with early RA. The primary end point, a composite of death from cardiovascular causes, non-fatal MI, non-fatal stroke and revascularisation, will be assessed after 5 years' of follow-up.

\section{Objective}

To evaluate the effect of a targeted, intensified, multidimensional intervention compared with conventional treatment of modifiable risk factors for CVD in patients with early RA.

\section{METHODS}

\section{Trial design}

The study is a prospective, randomised, open label trial, blinded end point outcome assessment ${ }^{22}$ with balanced randomisation (1:1) conducted in 10 outpatient clinics in Denmark. Follow-up visits for patients in the intervention group are scheduled to occur at baseline and then after 2, 4 and 12 weeks and thereafter every third month for 5 years after randomisation (see figure 1). The control group will be monitored for RA disease activity and comorbidity after 2, 4 and 12 weeks and thereafter following national guidelines for RA. ${ }^{23}$ Prevention of CVD risk factors in the control group will be treated in general practice according to national guidelines for diabetes (2011), hypertension (2009) and CVD (2013). ${ }^{24-26}$ A closeout visit will take place after study termination. Recruitment will begin in September 2014 and is scheduled to be completed at the end of September 2020.

\section{Participants}

The participants in the study have RA diagnosed by the treating rheumatologist.

Key inclusion criteria: RA according to the revised American College of Rheumatology (ACR) 2010 criteria $^{27}$ and plasma low-density lipoprotein (LDL) $>2.5 \mathrm{mmol} / \mathrm{L}$.

Exclusion criteria: Previous disease-modifying antirheumatic drug (DMARD) or oral glucocorticoid treatment, pregnant or lactating women, current infection with parvovirus B19, hepatitis B, hepatitis C or HIV or any condition contraindicating the study medication and a previous report of hospitalisation for myocardial ischaemia defined as follows: (1) non-fatal MI defined according to national and international guidelines, (2) acute coronary syndrome (ACS) including acute ischaemic symptoms with possible biomarker changes or ECG changes that do not meet the criteria for MI, (3) angina pectoris, (4) revascularisation (percutaneous coronary intervention (PCI) or coronary artery bypass grafting (CABG) (Box 1).

Other requirements for inclusion are willingness to participate for the duration of the trial. All patients will receive verbal and written information about the trial and sign a consent form before inclusion. The study nurse obtains written consent before inclusion and randomisation.

\section{Interventions}

At baseline, when the diagnosis of RA is established, blood samples for LDL, DMARD, glycated haemoglobin (HbAlc) and urine for microalbuminuria will be routinely taken. After 2 weeks, patients will be invited to participate in the study if they have LDL $>2.5 \mathrm{mmol} / \mathrm{L}$ and none of the exclusion criteria.

After written consent is obtained, patients will be randomised to either receive (1) Intensified multidimensional intervention involving strict treatment goals 


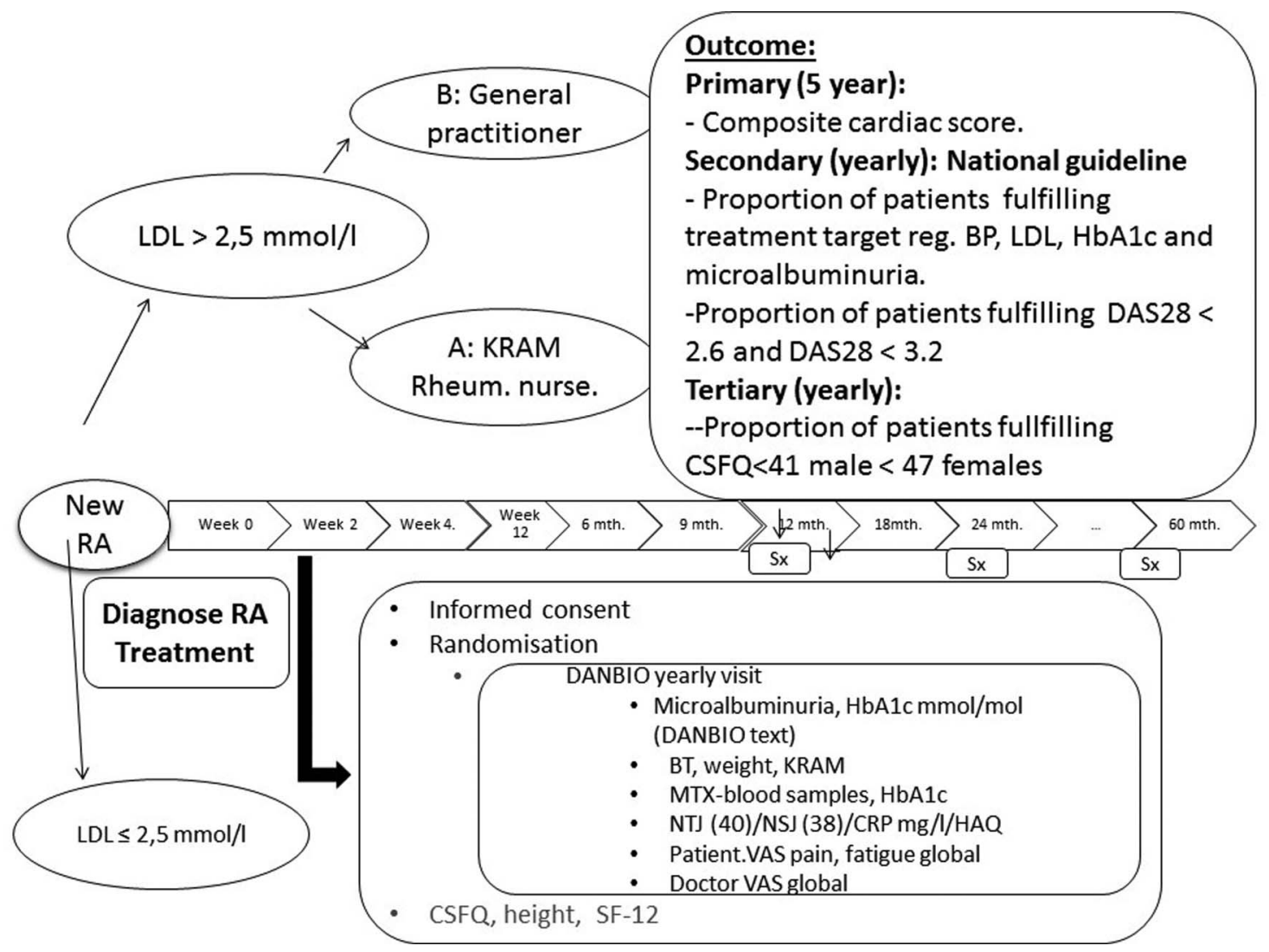

Figure 1 Trial design, visits and end points. BP, blood pressure; CRP, C reactive protein; CSFQ, Changes in Sexual Functioning Questionnaires; DAS28, disease activity score 28; HAQ, Health Assessment Questionnaire; HbA1c, glycated haemoglobin; LDL, low-density lipoprotein; RA, rheumatoid arthritis; VAS, visual analogue scale.

according to national guidelines or (2) Conventional treatment for multiple risk factors from their general practitioner (GP) according to the national guidelines for diabetes (2011), CVD (2013) and hypertension $(2009)^{24-26}$ with the possibility of being referred to specialists. The GP will receive an electronic notification to treat diabetes, hypertension or CVD if present. The patient has to arrange the appointment with the GP. The GP does not receive information about the ERACORI study protocol or the study hypothesis, but is of course free to search for the study in http://www. clinicaltrials.gov.All patients will fill out the short form 12-items health survey (SF-12) and Changes in Sexual Functioning Questionnaires (CSFQ), and disease activity score C-reactive protein (DAS28-CRP) will be registered using the Danish Danbio registry. LDL, DMARD, HbAlc and microalbuminuria will be measured.

1. In the intervention group, all patients will receive $40 \mathrm{mg}$ simvastatin according to national guidelines. Stepwise introduction of pharmacological therapy targeting (1) hyperlipidaemia, (2) hypertension, (3) hyperglycaemia and (4) microalbuminuria and behaviour modification will be controlled by the project team. (1)Patients with adverse effects to firstline statin will be treated with $80 \mathrm{mg}$ atorvastatin and subsequently treated with ezetimibe $10 \mathrm{mg}$ if side effects to atorvastatin are seen. ${ }^{28}$ Patients whose hyperlipidaemia is subsequently judged by their physician to require additional lipid-lowering therapy or treatment could be added to trial treatment. The target is LDL cholesterol $<2.5 \mathrm{mmol} / \mathrm{L}^{25}$

2. At each follow-up visit, antihypertensive drug therapy will be titrated to achieve target blood pressures $(<140-90 \mathrm{~mm} \mathrm{Hg})$ for patients without diabetes and $(<130-80 \mathrm{~mm} \mathrm{Hg})$ for patients with diabetes. ${ }^{24} 25$ The primary drug of choice is an angiotensin-II receptor antagonist at the maximal recommended dose if tolerated. All patients with microalbuminuria (urinary albumin creatinine ratio $30-299 \mathrm{mg} / \mathrm{g}$ ) ${ }^{29} 30$ will be treated with an angiotensin-II receptor antagonist if other causes, for example, urinary tract infection, can be ruled out. Combination treatment with an angiotensin-II receptor antagonist and an ACE inhibitor or a renin inhibitor will not be advised.

3. HbAlc levels above $48 \mathrm{mmol} / \mathrm{mol}(6.5 \%)$ will be treated with metformin. ${ }^{25}$

4. Patients in the intervention group have in-person contact with the study team every third month with response on LDL cholesterol, HBA1c and blood pressure. Every 6 months, a counselling session regarding diet, smoking, alcohol use and exercise habits is provided by the study nurse. Additional phone calls between the scheduled visits if further counselling regarding treatment is required (figure 2). 


\section{Box 1 Eligibility criteria at a glance}

\section{Inclusion}

- Age $>18$ years

- Early rheumatoid arthritis (American College of Rheumatology 2010 criteria)

- Plasma low-density lipoprotein $>2.5 \mathrm{mmol} / \mathrm{L}$

Disease-modifying antirheumatic drug (DMARD) Naïve

- Steroid Naïve

Exclusion

Pregnancy

- Lactation

- Ongoing/previous DMARD therapy

- Ongoing/previous steroid therapy

- Contraindications to any of the trial drugs

- Current infection with parvovirus B19, hepatitis B, hepatitis C or human immune deficiency virus. Previous report of hospitalisation for myocardial ischaemia defined as follows: (1) nonfatal myocardial infarction (MI) defined according to national and international guidelines, (2) acute coronary syndrome including acute ischaemic symptoms with possible biomarker changes or electrocardiographic changes that do not meet the criteria for MI, (3) angina pectoris, (4) revascularisation (percutaneous coronary intervention or coronary artery bypass grafting).

Behavioural change and behavioural risk factors for CVD (KRAM): Lifestyle recommendations according to the European Heart Society ${ }^{3132}$ are no smoking, weight reduction if body mass index $\left(\mathrm{BMI} \geq 25 \mathrm{~kg} / \mathrm{m}^{2}\right)$, especially if $\mathrm{BMI} \geq 30 \mathrm{~kg} / \mathrm{m}^{2}, 30 \mathrm{~min}$ of moderately vigorous exercise on most days of the week and a healthy diet. Stopping smoking is encouraged in all smokers. Nicotine chewing gum and transdermal nicotine patches have been widely used in helping quitters to go through the initial difficult weeks/months of smoking cessation, and will be recommended to all smokers.

Physical activity with vigorous exercise on most days of the week will be recommended. Also recommended will be a healthy diet that includes a wide variety of foods and energy intake adjusted to avoid overweight. The diet should consist of fruits and vegetables, wholegrain cereals and bread, fish (especially oily), lean meat, low fat dairy products and replacement of saturated fat with monounsaturated and polyunsaturated fats. Hypertensive patients should reduce salt intake. The Danish National Board of Health advises no more than 14 units of alcohol per week for men and 7 units per week for women. ${ }^{33}$

Treatment algorithm RA: The start dose of oral methotrexate is $15 \mathrm{mg}$ /week, increasing the dose by $5 \mathrm{mg}$ every second week to $25 \mathrm{mg}$ after 4 weeks. If a swollen joint is observed or at any other later visit, it is injected with glucocorticoids (kenalog-trimacinalone). If a swollen joint is observed after 6 weeks or at any later visit, daily orally $200 \mathrm{mg}$ hydroxychloroquine and $2000 \mathrm{mg}$ SalazopyrinEntabs will be added and orally methotrexate changed to $25 \mathrm{mg}$ subcutaneously/week. If DAS28-CRP <2.6 at year 1 and no swollen joints are observed, treatment will be tapered to $15 \mathrm{mg}$ methotrexate weekly. If unacceptable side effects occur, the route

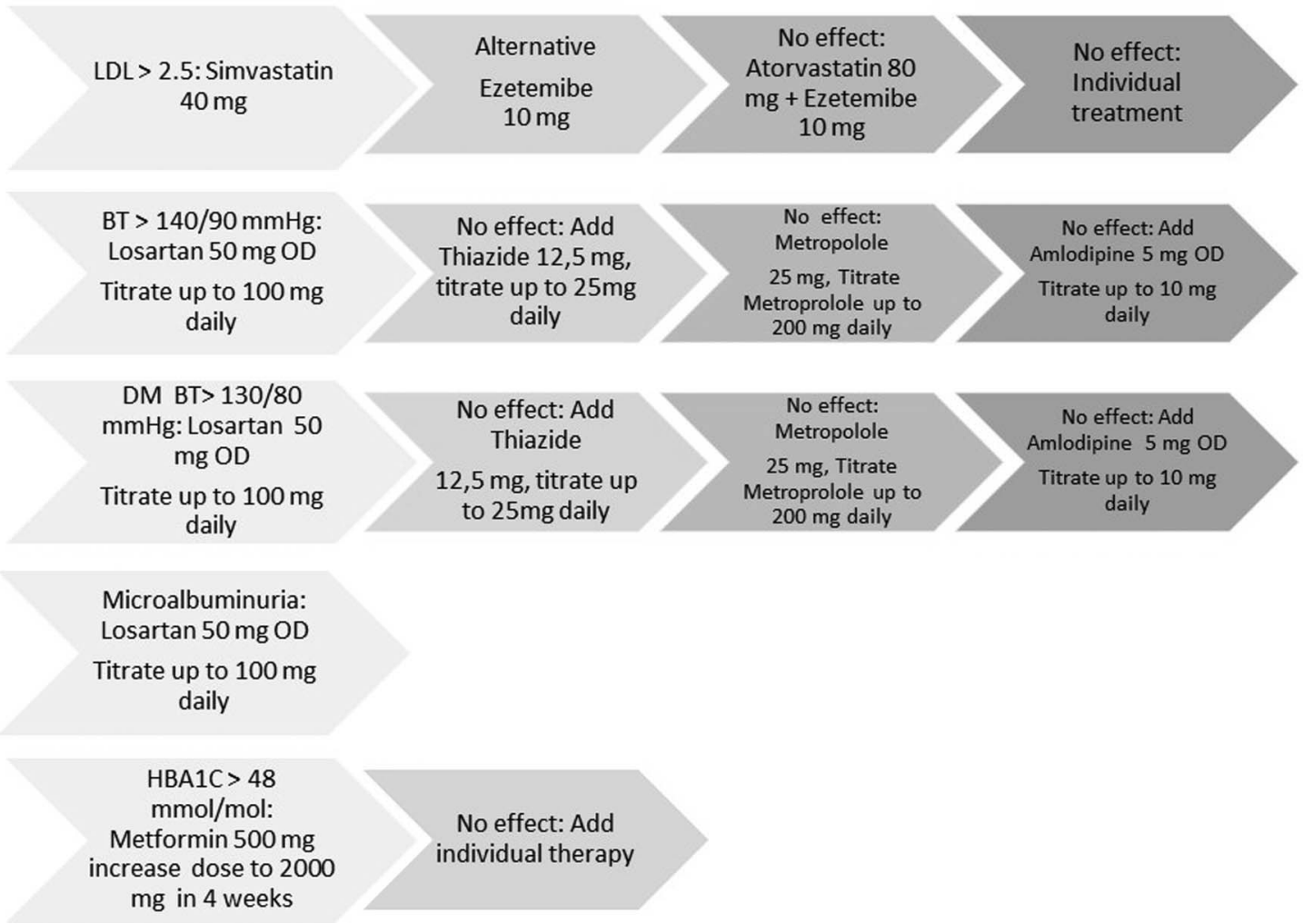

Figure 2 Cardiovascular treatment algorithm—Stepwise Guideline Intervention ERACORI Study. 
of administration can be changed to subcutaneous methotrexate at the same dose. The dose escalation should be guided by efficacy and/or adverse events. See figure 3. Furthermore, swollen joints can be injected with glucocorticosteroids according to the chart (maximum 4 joints or $4 \mathrm{~mL}$ per visit, according to the CIMESTRA algorithm previously published. ${ }^{34}$ If subsequently, evaluated in a 3-monthly setting, disease activity preceded higher than DAS28-CRP 3.2, initiation and treatment with biologics will be applied according to national guidelines. ${ }^{23}$ Oral glucocorticoids will not be allowed.

All patients will receive folic acid as well as calcium and vitamin D supplementation in accordance with national guidelines.

The ERACORI programme will be administered similarly at all sites. All study sites are included after a meeting with the principal investigator, the co-investigator, the local investigator and their study team. The purpose is to discuss the study and the practical aspects. Additional meetings will be arranged when necessary. Phone calls and emails are used to clear day-to-day questions with the co-investigator or principal investigator. All study sites are provided with an ERACORI trial site file. All included patients have a file with a checklist of scheduling patient visits for the physician or nurse, when to control blood tests (week -2 and to 60 months). There is a checklist for all patient visits stating what is supposed to take place at every visit. Patient information, informed consent file and the relevant questionnaires in hard copy for all study visits are included as well. Data are registered in the Danish Danbio Registry. All investigators are entering data on the study participants into the same module using individual usernames, passwords and user rights. This makes it possible for the co-investigator once a year to audit if all information is entered as planned in the Danbio Registry.

\section{Outcomes}

The primary outcome: The primary cardiovascular disease end point will be a composite measure of death from cardiovascular causes, non-fatal MI, non-fatal stroke and cardiac revascularisation after 5 years' of follow-up.

The event committee will adjudicate all deaths from any cause. Death will be classified as either cardiovascular or non-cardiovascular as illustrated in figure 4 . If no non-cardiovascular cause can be identified, the cause of death will always be considered as being cardiovascular. Cardiovascular deaths will be further classified in relation to time as either sudden or non-sudden. Sudden

\section{LDL>2.5 - Early RA intervention algorithm}

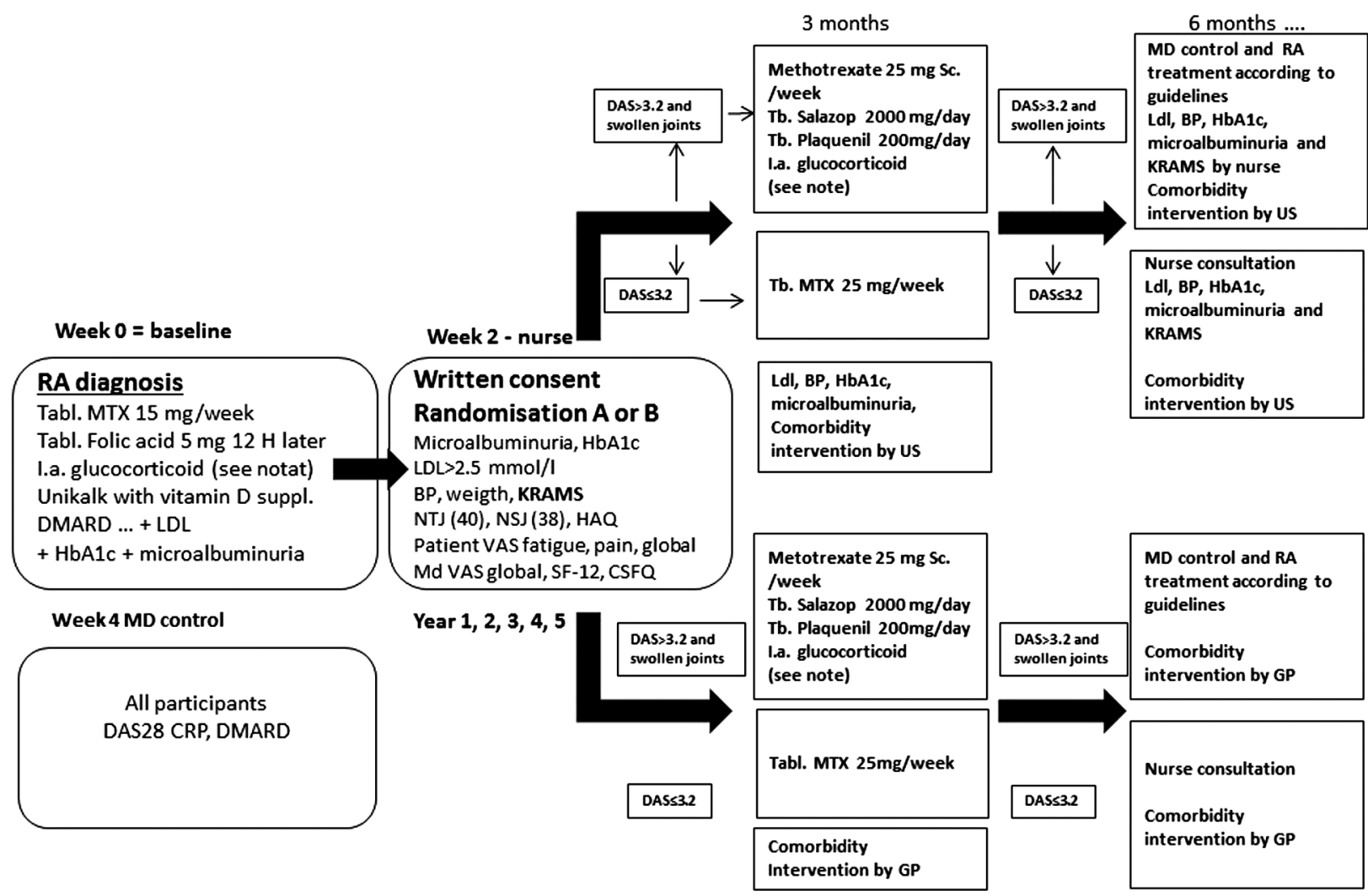

Figure 3 Early RA intervention algorithm. BP, blood pressure; CRP, C-reactive protein; CSFQ, Changes in Sexual Functioning Questionnaires; DAS28, disease activity score 28; DMARD, disease-modifying antirheumatic drug; GP, general practitioner; $\mathrm{HAQ}$, Health Assessment Questionnaire; HbA1c, glycated haemoglobin; LDL, low-density lipoprotein; MTX, methotrexate; RA, rheumatoid arthritis; SF-12, short form 12-items health survey; VAS, visual analogue scale. 


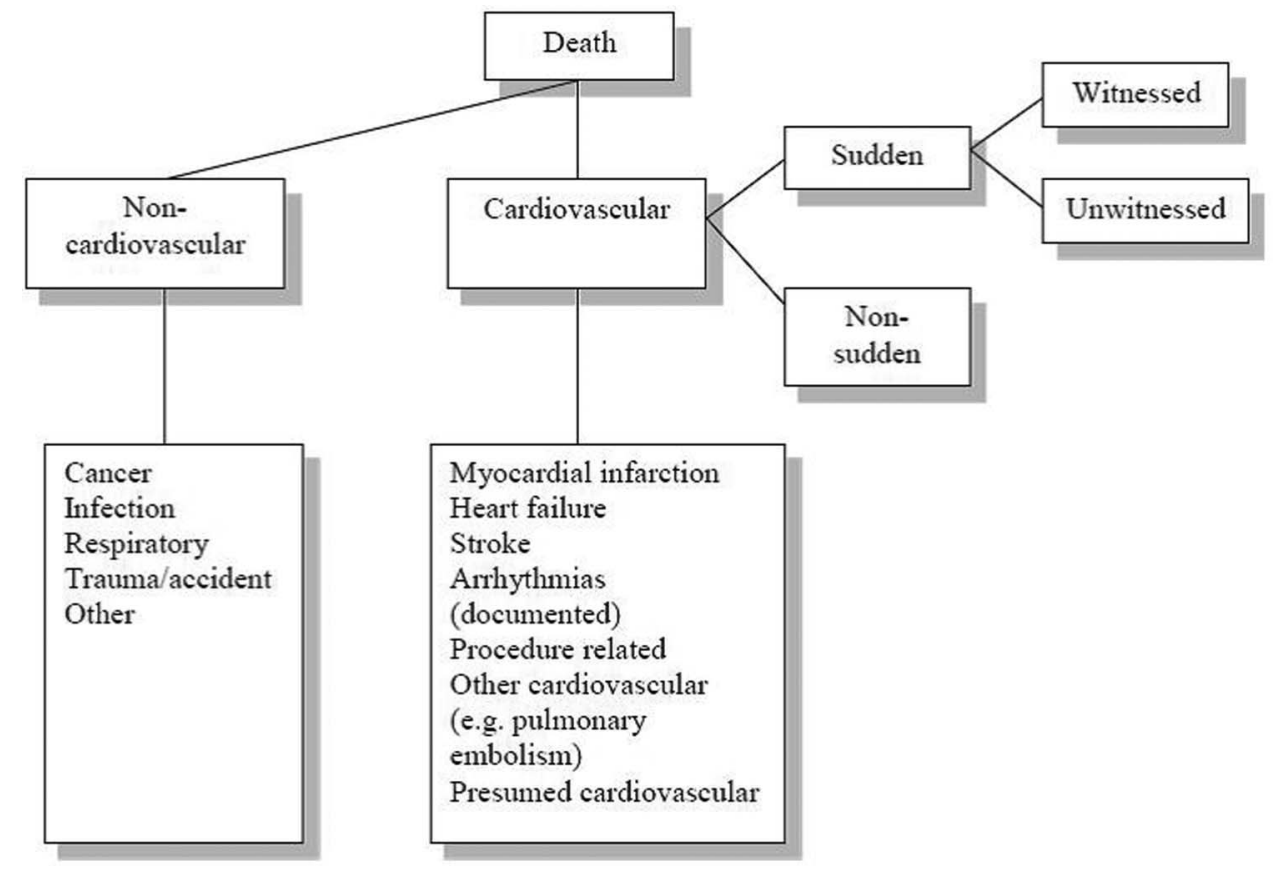

Figure 4 Classification of death in the trial.

deaths are described as either (1) witnessed and instantaneous or occurring within $1 \mathrm{~h}$ of new symptoms or (2) unwitnessed with no apparent cause (found dead). The remaining cardiovascular deaths will be classified as non-sudden.

Cardiovascular deaths will be subclassified as caused by (1) MI, (2) heart failure, (3) stroke, (4) documented arrhythmia, (5) procedure-related, (6) other cardiovascular causes including pulmonary embolism or (7) presumed cardiovascular death.

1. Death due to MI is defined as a primary fatal event that occurs within 7 days of an MI documented by autopsy or an MI defined according to national and international guidelines.

2. Death due to heart failure is defined as death occurring after a period of increasing symptoms and signs of heart failure.

3. Death due to stroke is defined as development of acute severe neurological deficit with or without documentation by CT scan. Deaths occurring within 2 weeks of a stroke where no other competing causes can be identified are classified as death due to stroke. Death from stroke occurring as a direct consequence of an investigation/procedure/operation will be classified as procedure-related death.

4. Death due to documented arrhygmia as primary cause of death.

5. Death due to procedure-related death is defined as death following a cardiovascular investigation/procedure/operation within $24 \mathrm{~h}$.

6. Death due to other cardiovascular causes is death occurring after other cardiovascular events like, for example, pulmonary embolism, ruptured aortic aneurysm, etc.
7. Presumed cardiovascular death is all deaths not attributed to the above categories of cardiovascular deaths or not attributed to a documented noncardiovascular cause. This category includes deaths from unknown cause.

Non-cardiovascular death will be classified as one caused by cancer, primary infectious disease, respiratory disease, trauma/accident, suicide or other causes for non-cardiovascular death as illustrated in figure 4.

The adjudication for cause of death, non-fatal MI, non-fatal stroke and cardiac revascularisation will be based on information from patient records, the death certificate and information from the GP. Two members of the event committee will adjudicate each event separately. In case of disagreement between the two members of the event committee, there will be a meeting between these two members and the chairman of the committee will make a decision in each case.

Both members of the event committee will be blinded to patient treatment allocation.

Secondary outcome: Secondary outcomes are the proportion of patients achieving LDL cholesterol $<2.5 \mathrm{mmol} / \mathrm{L}$, HbAlc $<48 \mathrm{mmol} / \mathrm{mol}$ (HbAlc $<6.5 \%$ ), blood pressure $<140 / 90 \mathrm{~mm} \mathrm{Hg}$ for patients without diabetes and $<130 / 80 \mathrm{~mm} \mathrm{Hg}$ for patients with diabetes and normoalbuminuria (urinary albumin creatinine ratio $<30 \mathrm{mg} / \mathrm{g}$ ) after 1-year of follow-up ${ }^{24-26}$; this is in agreement with present national guidelines, which will be adjusted according to any future changes in the respective national guidelines. Secondary outcomes are the proportion of patients in each group achieving low RA disease activity after 1-year: DAS28-CRP $<3.2$ and DAS28-CRP $<2.6$ at 12, 24 and 60 months. Furthermore, 
all to hospitalisations will be adjudicated by the event committee and hospitalisation will be defined as

1. Hospitalisation for acute medical reasons;

2. Hospitalisation for planned, elective reasons.

Hospitalisations for acute medical reasons are classified as cardiovascular or non-cardiovascular. An acute hospitalisation is defined as non-planned and must include a stay in hospital for at least one night, that is, a minimum covering two consecutive dates and accompanied by a calendar date change. This classification will be based on the total findings from the whole hospital admission. Cardiovascular acute hospitalisations are further classified as due to (1) worsening heart failure, (2) myocardial ischaemia, (3) arrhythmia, (4) stroke, (5) other cardiovascular hospitalisations or (6) presumed cardiovascular hospitalisation. The classification is as follows:

1. Hospitalisation for worsening heart failure is defined as a non-planned admission to hospital lasting at least one night and presenting symptoms or signs related to congestive heart failure. Furthermore, objective findings and an increase in congestive heart failure treatment will be evaluated.

2. Hospitalisation for myocardial ischaemia is defined as follows:

a. Non-fatal MI is defined according to national and international guidelines.

b. ACS including acute ischaemic symptoms with possible biomarker changes or electrocardiographic changes which do not meet the criteria for MI.

c. Angina pectoris.

d. Revascularisation (PCI or CABG).
3. Hospitalisation due to supraventricular and ventricular arrhythmias.

4. Hospitalisation for stroke is defined as haemorrhagic and ischaemic stokes and transient ischaemia attacks.

5. Hospitalisation for other cardiovascular reasons such as pulmonary embolism or ruptured aortic aneurism.

6. Hospitalisation for a presumed cardiovascular reason is defined as no non-cardiovascular cause identified.

Non-cardiovascular hospitalisation for acute medical reasons is classified as due to cancer, infection, respiratory disease, trauma/accident, suicide or other causes.

Hospitalisation for planned, elective reason is divided into cardiovascular or non-cardiovascular, and cardiovascular hospitalisations are further divided into hospitalisation for myocardial ischaemia, arrhythmias, heart failure or other cardiovascular hospitalisations. Non-cardiovascular hospitalisation for planned reasons is not further subdivided (figure 5). ${ }^{35-37}$

All hospitalisations will be adjudicated by the event committee as illustrated in figure 5 .

Secondary outcomes defined as described above are evaluated at 12, 24 and 60 months.

Tertiary outcome: Sexual function: The sexual functioning of the patients is measured by the validated, gender-specific 14-item Changes in Sexual Functioning Questionnaire (CSFQ) ${ }^{38}$ In addition, 14 explorative questions concerning sexual well-being, body image and sexological counselling will be asked. In order to obtain representative control data, a portion of these questions is duplicated from The National Health

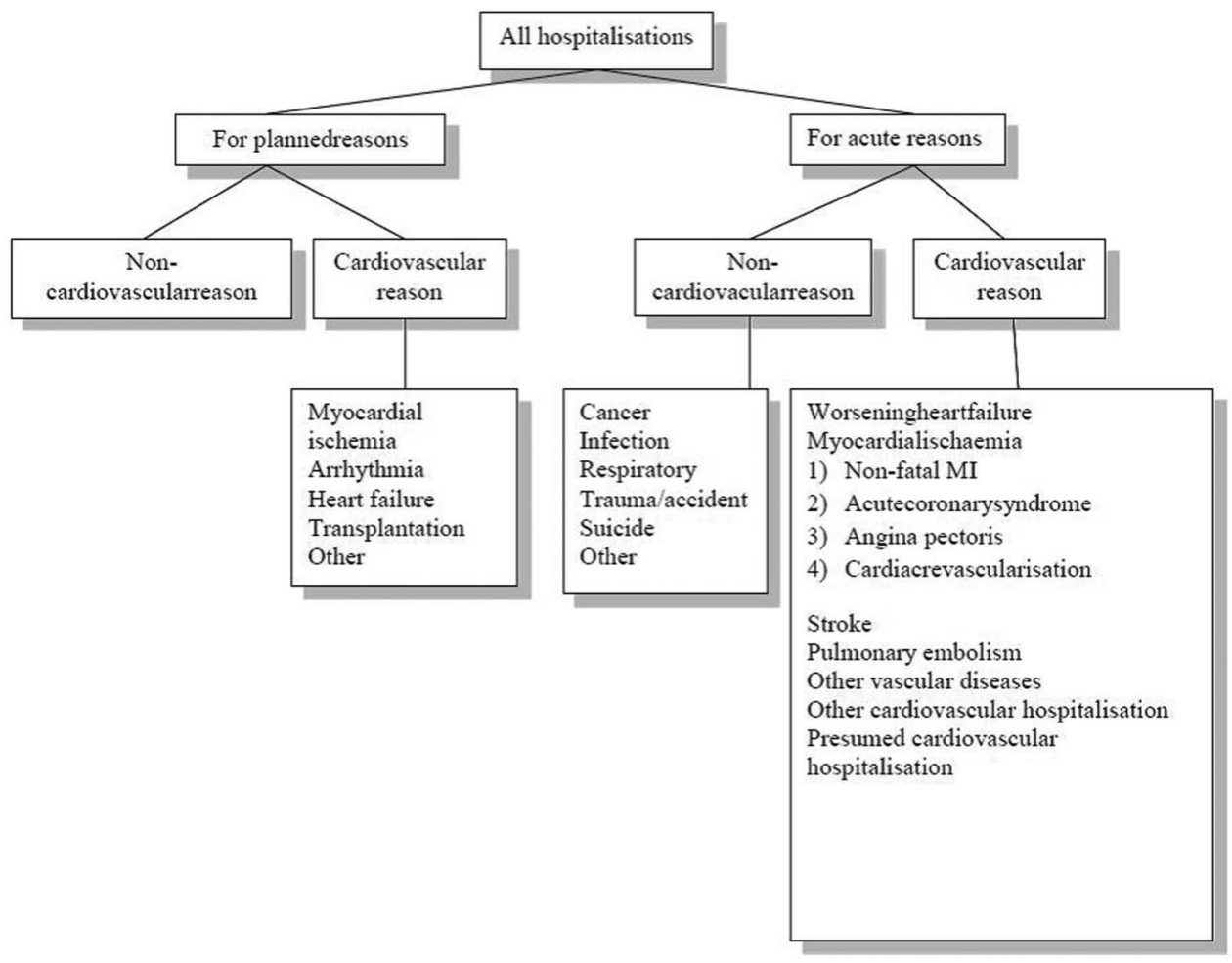

Figure 5 Classification of hospitalisation for planned or acute reasons in the trial. MI, myocardial infarction. 
Interview Surveys (SUSY). ${ }^{39}$ Finally, to avoid confounding, patients will be screened for symptoms of major depression using the 10-item Major Depression Inventory (MDI). ${ }^{40}$

Measurements: The patients will be seen at predefined intervals of 2, 4 and12 weeks and thereafter every third month for 5 years. In addition, the participants will be registered in the Danbio registry at every visit. Disease activity will be measured by disease activity score calculated on 28 joints (DAS28-CRP).

Disability status will be self-reported according to the Danish-validated version of the Stanford Health Assessment Questionnaire (HAQ). Patient-estimated global health, pain and fatigue will be assessed on a visual analogue scale (VAS) $0-100 \mathrm{~mm} .{ }^{41}$ Self-reported health status will be measured by SF-12 health perception scales; scores range from 0 to 100 , with higher scores representing better health. ${ }^{42}{ }^{43}$ Body weight and height will be measured at baseline and BMI calculated as $\mathrm{BMI}=$ weight/height squared $\left(\mathrm{kg} / \mathrm{m}^{2}\right)$. Smoking is defined as daily smoking, current or past smoking and never smoking. ${ }^{44}$
A cumulated dose of intra-articular glucocorticosteroid administered between baseline and after 1-year will be registered. Blood samples will be taken to measure $\mathrm{C}$ reactive protein (CRP), serological markers (IG-M rheumatoid factor), anticyclic citrullinated peptide antibodies (anti-CCP) and lipid status. Double reading of the systolic and diastolic blood pressure will be obtained on the right arm with the participant in a sitting position after a $5 \mathrm{~min}$ rest with two measurements with 1-2 min between them. ${ }^{45}$ An overview of trial design, visits and end points is shown in table 1.

\section{Safety}

It is the investigator's responsibility to ensure that all serious adverse reactions/adverse events (AE) are immediately reported to the sponsor and the project leader, who are responsible for notifying the regional biomedical research ethics committee. Reports to the regional Biomedical Research Ethics Committee must be accompanied by comments on the possible consequences for the trial. The project leader is also responsible for informing the participating departments of what a

Table 1 Trial design, visits and end points

\begin{tabular}{|c|c|c|c|c|}
\hline Variable & Baseline & 12 months & 24 months & 60 months \\
\hline Sex & $x$ & & & \\
\hline Age & $x$ & & & \\
\hline Height & $x$ & & & \\
\hline Current smoker & $x$ & $X^{1}$ & $x^{2}$ & $x^{3}$ \\
\hline Tender joints & $x$ & $X^{1}$ & $x^{2}$ & $x^{3}$ \\
\hline Swollen joints & $x$ & $X^{1}$ & $x^{2}$ & $x^{3}$ \\
\hline IgM-RF & $x$ & & & \\
\hline Anti-CCP & $x$ & & & $x^{4}$ \\
\hline $\mathrm{C}$ reactive protein & $x$ & $X^{1}$ & $x^{2}$ & $x^{3}$ \\
\hline Patient global & $x$ & $x^{1}$ & $x^{2}$ & $x^{3}$ \\
\hline Patient pain & $x$ & $X^{1}$ & $x^{2}$ & $x^{3}$ \\
\hline Patient fatigue & $x$ & $X^{1}$ & $x^{2}$ & $x^{3}$ \\
\hline LDL cholesterol & $x$ & $x^{1}$ & $x^{2}$ & $x^{3}$ \\
\hline HDL cholesterol & $x$ & $x^{1}$ & $x^{2}$ & $x^{3}$ \\
\hline Total cholesterol & $x$ & $x^{1}$ & $x^{2}$ & $x^{3}$ \\
\hline Triglycerides & $x$ & $x^{1}$ & $x^{2}$ & $x^{3}$ \\
\hline Body weight & $x$ & $X^{1}$ & $x^{2}$ & $x^{3}$ \\
\hline Blood pressure: systolic & $x$ & $x^{1}$ & $x^{2}$ & $x^{3}$ \\
\hline Blood pressure: diastolic & $x$ & $x^{1}$ & $x^{2}$ & $x^{3}$ \\
\hline Cardiovascular acute hospitalisation & $x$ & $x^{1}$ & $x^{2}$ & $x^{3}$ \\
\hline Cardiovascular disease & $x$ & $x^{1}$ & $x^{2}$ & $x^{3}$ \\
\hline CSFQ-14 & $x$ & $X^{1}$ & $x^{2}$ & $x^{3}$ \\
\hline $\mathrm{MDI}$ & $x$ & $X^{1}$ & $x^{2}$ & $x^{3}$ \\
\hline SF-12 & $x$ & $x^{1}$ & $x^{2}$ & $x^{3}$ \\
\hline LI-Sat & $x$ & $X^{1}$ & $x^{2}$ & $x^{3}$ \\
\hline Self-reported 15-item questionnaire on sexual activity & $\mathrm{x}$ & $X^{1}$ & $x^{2}$ & $x^{3}$ \\
\hline
\end{tabular}

Paper 1. Multifactorial intervention and cardiovascular disease in patients with early RA after a 1-year follow-up considering data marked with (1). Paper 2. Multifactorial intervention and cardiovascular disease in patients with early RA after a 2-year follow-up considering data marked with (2). Paper 3. Effect of a multifactorial intervention on mortality in patients with RA after 5 years of follow-up considering data marked with (3). CCP, cyclic citrullinated peptide antibodies; CSFQ, Changes in Sexual Functioning Questionnaires; HDL, high-density lipoprotein; KRAMS nurse counselling regarding diet, smoking, alcohol use and exercise habits; LDL, low-density lipoprotein; Li-sat, Questions concerning sexual well-being, body image and sexological counselling; MDI, Major Depression Inventory; NSJ, number of swollen joints; NTJ, number of tender joints; RA, rheumatoid arthritis; RF, rheumatoid factor SF-12, short form 12-items health survey. 
serious adverse reaction/adverse event entails. It is also the responsibility of the project leader to submit on an annual basis, starting 1 year after approval of the study, a list of serious unexpected adverse reactions that have occurred in the period to the regional Biomedical Research Ethics Committee. Standard AE and serious adverse events will be systematically registered and reported to the Danish Health and Medicines Authority. The participants in the trial are treated in accordance with national guidelines. Those patients who suffer harm from trial participation will be referred to the patient compensation association.

\section{Randomisation}

After the baseline assessment, the participants will be randomly assigned to either the control group or the intervention group. The randomisation sequence is created using SAS (SAS V. 9.2) statistical software and is stratified by centre with a 1:1 allocation using random block sizes of 2, 4 and 6 . The allocation sequence will be concealed from the researcher enrolling and assessing participants in sequentially numbered, opaque and sealed envelopes. Aluminium foil inside the envelope will be used to render the envelope impermeable to intense light. After revealing the content of the envelope, both patients and the treating rheumatologists are aware of the allocation and the corresponding treatment. Outcome assessors and data analysts will, however, be kept blinded. Prior to the outcome assessments, the patients will be asked by the research assistant not to mention the treatment to which they have been allocated.

\section{Blinding}

After assignment of the participants to the intervention, the senior cardiologist (event committee) is blinded when validating the cardiovascular end points after the 1-year, 2-year and 5-year follow-ups.

\section{Data collection, management and confidentiality}

All primary analyses will be performed on an intention-to-treat basis; all patients randomised will be included in the primary analyses. Study participation is considered to be complete for any individual participant at the time he or she had an occurrence of the primary end point, had informed consent withdrawn, was unable to be followed further because the study site closed, or had been followed for at least September 2020. The exposure time was calculated as the time between randomisation and the first major cardiovascular event, the date of death, the date of the last study visit, the date of withdrawal or loss to follow-up, or September 2020, whichever came first. Regarding confidentiality, all data will be electronically stored in electronic clinical databases approved by the Danish health authorities, which approved the procedure. The patient consent form and the SUSY questionnaire is stored in paper form and kept locked and at the investigator's site.
Statistical methods

\section{Sample size and power considerations}

Assuming a constant rate of events of $10 \%$ per year, among patients with RA allocated to the control group, and a risk reduction with the ERACOR programme corresponding to $50 \%$, it was decided to include a total of 300 patients with RA (150 patients in each group). For a comparison of two independent binomial proportions using Pearson's $\chi^{2}$ statistic with a $\chi^{2}$ approximation with a two-sided significance level of $0.05(\mathrm{p}<0.05)$, a sample size of 118 per group achieves a power of at least $90 \%$ of patients having a composite of death from cardiovascular causes, non-fatal myocardioinfarction, non-fatal stroke and cardiac revascularisation are $45 \%$ and $25 \%$. As illustrated in table 2, with 150 patients in each group included in the intention-to-treat (ITT) population, there is a reasonable statistical power $(83 \%)$ even to detect a difference between $25 \%$ and $12 \%$ of patients having an event during the five years of observation.

\section{Prespecification of the analysis}

The primary cardiovascular disease end point is a composite measure of death from cardiovascular causes, non-fatal MI, non-fatal stroke and cardiac revascularisation after a 5-year follow-up. The primary end point is a binary outcome, enabling interpretation from the relative benefit and harm from the 'ERACORI programme' and, on the basis of the observed events in the control group, we can communicate absolute benefit and harm as well.

The primary end point (60 months from baseline) will be analysed with 'survival analysis' according to the ITT principle, with event curves for the time to the first event based on the Kaplan-Meier analysis and treatments being compared with the use of the log-rank test. A Cox regression model is used to calculate the HR for the primary end point. For the purpose of sensitivity, the analyses will be adjusted for age, the duration of RA, sex, clinical centre and cardiovascular status at baseline.

Binary outcomes: Logistic regression analysis and $\chi^{2}$ tests will be used to compare categorical variables. Comparison between dichotomous outcome variables will be presented as risk ratios (RRs) with $95 \%$ CIs, and

\begin{tabular}{llll} 
Table 2 & Power calculation on different CVD prevalences \\
\hline Scenario & $\begin{array}{l}\text { Proportion } \\
\text { (Control) }\end{array}$ & $\begin{array}{l}\text { Proportion } \\
\text { (ERACORI) }\end{array}$ & $\begin{array}{l}\text { Statistical } \\
\text { power* }\end{array}$ \\
\hline 1 & 0.50 & 0.25 & 0.995 \\
2 & 0.50 & 0.30 & 0.946 \\
3 & 0.50 & 0.35 & 0.750 \\
4 & 0.25 & 0.10 & 0.932 \\
5 & 0.25 & 0.12 & 0.830 \\
6 & 0.25 & 0.15 & 0.582 \\
\hline${ }^{*} \chi^{2}$ approximation with a two-sided significance level of 0.05, with \\
a sample size of 150 patients with RA per group. \\
CVD, cardiovascular diseases; RA, rheumatoid arthritis.
\end{tabular}


interpreted on the basis of the absolute benefit or harm via risk differences (RDs) with 95\% CIs. The sensitivity analyses will be modelled using logistic regression analysis to investigate the relationship between these discrete responses and a set of potentially confounding variables. The logistic model is based on a General Linear Model (GLM): a function of the mean of the response variable is assumed to be linearly related to the explanatory variables. The model will include treatment and centre status as fixed effects, with the baseline value (if assessed) of the relevant variable as a covariate.

Continuous outcomes: Measured variables are compared by means of analysis of covariance, with baseline values as covariates to adjust for differences between the groups at randomisation. This analysis of covariance will be modelled using the method of least squares to fit a GLM. The sensitivity analyses for the continuous outcomes will be modelled using GLM. GLM handles models relating one or several continuous dependent variables to one or several independent variables. As for the binary outcomes, the model will include treatment and centre status as fixed effects, with the baseline value (if assessed) of the relevant variable as a covariate.

\section{Full analysis set}

The primary analyses will be performed according to the ITT principle. The ITT principle implies that the primary analysis should include all randomised participants. Compliance with this principle would necessitate a complete follow-up of all randomised participants for study outcomes. In practice, this ideal may be difficult to achieve for different reasons that will not necessarily introduce bias into the subsequent statistical analysis. In this study, the term 'full analysis set' is used to describe the analysis set, which is as complete as possible and as close as possible to the ITT ideal of including all randomised participants (ie, the ITT population). Preservation of the initial randomisation in analysis is important in preventing bias and in providing a secure foundation for statistical tests. The full analysis set provides a conservative strategy. Under many circumstances, it may also provide estimates of treatment effects which are more likely to mirror those observed in subsequent practice.

When data are incomplete at end point, we will perform sensitivity analyses based on an imputation of missing values using two different approaches: (1) multiple imputation with age, the duration of RA, sex, clinical centre and cardiovascular status at baseline entered as predictor variables and (2) imputation of 'best' and 'worst' case scenarios by replacing missing values with 'good' outcomes in one group and 'bad' outcomes in the other group. ${ }^{46}$ Where complete cases and different imputation techniques give different results, we will attempt to understand why and report this in the publications.
Per protocol set

The 'per protocol' set of participants is defined as a subset of the participants in the full analysis set who are more compliant with the protocol, characterised by the following criteria: Participants are defined as per protocol if they have no more than one absence from a clinical visit per year. One year in the analysis is defined as 12 months (plus/minus 1 month).

\section{Subgroups, interactions and covariates}

Subgroup analysis will be performed regarding participants with diabetes type II, obese $B M I \geq 30 \mathrm{~kg} / \mathrm{m}^{2}$, Smokers and seropositive patients, that is, positive anti-CCP and/or positive IgM RF, and high disease activity (DAS28CRp >3.2 after 6 months of treatment).

\section{Data monitoring}

The ERACORI study is carried out in accordance with the approved protocol and the applicable regulatory requirements and legislations in this field.

The role of the data monitoring committee (DMC) is to register death from cardiovascular causes, non-fatal MI, non-fatal stroke and cardiovascular revascularisation and death by any cause after $1,2,5$ and 10 years of follow-up. Two authors (BBL and ALS) will go through all patient records and register these data. In case of any discrepancies identified, TE will make the final decision. DMC is independent from competing interests.

In case the DMC is registering a $50 \%$ higher mortality rate from cardiovascular causes or a $50 \%$ higher prevalence of cardiovascular events after year one or year two of follow-up in any of the study groups, the principal investigator (TE) and the co-investigator (ALS) (The trial management committee) are responsible for informing the steering committee about the matter. The trial management committee makes the final decision to terminate the trial.

\section{ETHICS, DISSEMINATION, AUDITING AND DISCLOSURE}

All patients must give their written informed consent. The protocol is approved by the local ethics committee (DK S-2014007) and the Danish Health and Medicines Authority and performed in accordance with the Declaration of Helsinki ${ }^{47}$ and Oviedo. A report will be submitted to the ethics committee yearly; in case of changes in the Danish national guidelines for the comorbidity strategy, the ethics committee will be contacted. The trial is registered at Clinical Trials.Gov (NCT 02246257).

Auditing: The Danish national health authorities as well as the scientific committee do not require auditing for this specific study.

The scientific integrity of the project requires that the data from the ERACORI trial sites will be analysed in its entity. Thus, an individual centre is not supposed to report data collected from this centre alone. All abstracts and publications are expected to protect the integrity of 
the major objective of the study. The recommendations considering the timing and presentation of end point data and the meetings at which they might be presented will be given by the steering committee. The review process in each paper or abstract will be distributed and reviewed by all principal investigators in the study prior to submission to a peer-reviewed journal or national or international conference. The study results will be released to the participating physicians, patients and the general medical community.

Two internal monitors will ensure that all data have been entered correctly and in accordance with the protocol. If data are missing, queries will be sent out electronically to the study sites. Disclosures for all the authors are accessible at the Danish Health and Medicines Authority in the updated version.

\section{DISCUSSION}

The ERACORI study is expected to contribute significant new knowledge about CVD prevention strategies in early patients with RA, thereby ensuring optimal treatment for CVD risk factors in this high-risk population.

The ERACORI intervention is a multidimensional intervention. We know that it is not possible to clear out which part is most efficient. Multidimensional interventions are in general considered more efficient, especially in CVD protection. Multifactorial risk reduction requires multifactorial intervention. Our study is inspired by the Steno 2 study in T2DM, which has shown remarkable CVD risk reduction by applying multifactorial intervention in a Danish high-risk population of patients with T2DM. Post-study evaluation of the results has indicated that most of the CVD benefit came from statin therapy, but it is clearly the combined intervention that is beneficial. ${ }^{20}$ In RA, it has further been evaluated in a meta-analysis this year that the antiinflammatory effect of statins in RA is significant and beneficial, resulting in lower disease activity in the 15 RA cohorts studied; ${ }^{48}$ that is why the secondary outcome evaluating disease activity was chosen. In the CARRE study on RA, both elevated systolic blood pressure and impaired renal function (measured as glomerular filtration rate (GFR)) were associated with increased risk of MI. ${ }^{49}$

Microalbuminuria is a somewhat overlooked CVD risk factor in a general population, despite being present in up to $7 \%$. In hypertensive patients, treatment and reduction in microalbuminuria is associated with improved CVD outcome, not to mention cases of patients with T2DM. Microalbuminuria is thought to reflect general vascular endothelial dysfunction, and as such a predictor of CVD. Since we think the RA population in this study is paralleled to a T2DM population in terms of CVD risk, we have included microalbuminuria as a risk factor. As mentioned above, even small declines in the GFR are predictive of MI in RA. ${ }^{49}$
We have chosen a design evaluating the effect of multifactorial intervention. As such, our study is designed to yield optimal clinical benefit, not to investigate and measure the effect of single risk factor intervention.

However, some may worry that the patients with RA in the control group might receive insufficient counselling compared to patients without RA and other high CVD risk groups. The GP receives an electronic patient-file notification that the patient is participating in the ERACORI protocol. Regarding patients in the control group, the GP will receive an electronic notification to treat diabetes, hypertension or CVD if present according to the guidelines. The patient has to arrange the appointment with the GP. The GP does not receive information about the ERACORI study protocol or the study hypothesis, but is of course free to search for the study in http://www.clinicaltrials.gov.

We are aware of the risk that patients might receive insufficient CVD counselling compared to patients without RA and other CVD high-risk groups. However, the aforementioned procedure is the standard care for CVD prevention and treatment in patients with RA in Denmark. At present, there is no tradition in the Danish rheumatology association to provide CVD prevention counselling as a standard along with the RA treatment. It is our hope that the results of the ERACORI study can contribute by showing how to make the most efficient strategy for CVD prevention in patients with early RA.

When we designed the intervention part of the ERACORI study we were inspired by the Steno II studies on patients with T2DM ${ }^{20} \quad 2150$ since the diabetes II population's CVD risk is similar to that of patients with RA. The choice of recommending simvastatin $40 \mathrm{mg}$ when LDL $>2,5 \mathrm{mmol} / \mathrm{L}$ is in line with the Danish guideline for cholesterol treatment of patients with T2DM. ${ }^{25}$ This is the reason why we have chosen not to follow the ESC guideline on this aspect.

Furthermore, it has been shown that ECS SCORE underestimates the CVD risk among patients with RA. ${ }^{51}$ In addition to a cholesterol lowering effect, simvastatin also has an anti-inflammatory effect which might be beneficial regarding prevention of CVD in patients with RA. ${ }^{52}$ The chronic inflammation and the endothelial dysfunction in patients with RA are not taken into account in the ERC guidelines SCORE risk estimation algorithm. We have used the ERC guidelines in designing the counselling regarding behavioural change and behavioural risk factors for CVD.

Since the CVD risk of the T2DM population is similar to that of patients with RA, we consider it relevant to include 300 patients with RA in the ERACORI study. ${ }^{20} 21$ In the steno II studies, it was possible with 166 patients with T2DM included to detect a significant difference in the CVD outcome between the two groups. The TRACE study was a single factor intervention study with $40 \mathrm{mg}$ atorvastatin versus placebo on patients with RA with 10 years' disease duration, which was prematurely 
terminated due to the CVD event rate in the $40 \mathrm{mg}$ atorvastatin group as well as in the placebo group. However, a higher number of patients are usually required when investigating single factor interventions compared to multidimensional interventions. ${ }^{53}$

If the ERACORI intervention programme is shown to be beneficial, implementation in daily clinical practice will be feasible.

A weakness is the non-blinded design. The rheumatologist and the patient were not blinded to the allocated intervention, which potentially could lead to bias. However, the outcome assessors of the cardiovascular end point were blinded to the patient intervention status, which has previously been shown to be a valid method. ${ }^{202150}$ To the best of our knowledge, this is the first RCT study to investigate the effect of an intensive multidimensional intervention aiming at preventing CVD among patients with early RA. The results of the study will be of importance for rheumatologists who treat patients with RA and can potentially change the way we monitor patients with RA and target treatment against CVD risk factors as well as RA in order to obtain better disease control and decrease the overall disease burden of our patients with RA with the possibility of improving survival.

\author{
Author affiliations \\ ${ }^{1}$ Department of Rheumatology, Copenhagen University Hospital, Bispebjerg \\ and Frederiksberg, Denmark \\ ${ }^{2}$ Musculoskeletal Statistics Unit, Department of Rheumatology, The Parker \\ Institute, Copenhagen University Hospital, Bispebjerg and Frederiksberg, \\ Denmark \\ ${ }^{3}$ Steno Diabetes Center, Gentofte, Denmark \\ ${ }^{4}$ Department of Cardiology, Aarhus University Hospital, Skejby, Denmark \\ ${ }^{5}$ Psychiatric Center, Sexological Clinic, Copenhagen University Hospital, \\ Denmark \\ ${ }^{6}$ Department of Clinical Medicine, Center for Sexology Research, Aalborg \\ University, Aalborg, Denmark \\ ${ }^{7}$ Department of Rheumatology, Diagnostic Centre, Regional Hospital \\ Silkeborg, Denmark \\ ${ }^{8}$ Department of Rheumatology, Svendborg Hospital, Svendborg, Denmark \\ ${ }^{9}$ Department of Rheumatology Sydvestjysk Sygehus, Esbjerg/Varde, Denmark \\ ${ }^{10}$ Reumaklinik Fyn, Odense, Denmark \\ ${ }^{11}$ Kong Christian X's Gigthospital i Gråsten, Denmark \\ ${ }^{12}$ Department of Rheumatology, Odense University Hospital, Odense, \\ Denmark \\ ${ }^{13}$ Reumaklinik, Roskilde, Denmark \\ ${ }^{14}$ Reumaklinik, Skanderborg, Denmark \\ ${ }^{15}$ Reumaklinik, Aabenraa, Denmark \\ ${ }^{16}$ Danbio National Registry, Glostrup University Hospital, Denmark
}

Acknowledgements Musculoskeletal Statistics Unit, The Parker Institute is supported by unrestricted grants from The Oak Foundation.

Contributors ALS, RC and TE conceived and developed the idea for the study. All authors contributed to the study design, writing of the first draft of the protocol, and revisions to the protocol paper. All authors will approve the final version of any paper before submission.

Funding Musculoskeletal Statistics Unit, The Parker Institute is supported by unrestricted grants from The Oak Foundation.

Competing interests None declared.

Provenance and peer review Not commissioned; externally peer reviewed.
Data sharing statement Data from the ERACORI trial will be stored in the Danish Data Archive (DDA) when data have been analysed and published. Through an agreement with the Danish Data Protection Agency, DDA preserves data materials containing personal identifiers. Data and the personal identifiers are stored separately and a special permit is required for access to the data. Data available on request for academic researchers.

Open Access This is an Open Access article distributed in accordance with the Creative Commons Attribution Non Commercial (CC BY-NC 4.0) license, which permits others to distribute, remix, adapt, build upon this work noncommercially, and license their derivative works on different terms, provided the original work is properly cited and the use is non-commercial. See: http:// creativecommons.org/licenses/by-nc/4.0/

\section{REFERENCES}

1. Semb AG, Kvien TK, Aastveit AH, et al. Lipids, myocardial infarction and ischaemic stroke in patients with rheumatoid arthritis in the Apolipoprotein-related Mortality RISk (AMORIS) Study. Ann Rheum Dis 2010;69:1996-2001.

2. Lindhardsen $\mathrm{JO}$, Ahlehoff $\mathrm{O}$, Gislason $\mathrm{GH}$, et al. The risk of myocardial infarction in rheumatoid arthritis and diabetes mellitus: a Danish nationwide cohort study. Ann Rheum Dis 2011;70:929-34.

3. De Vera MA, Choi H, Arhamowicz M, et al. Statin discontinuation and risk of acute myocardial infarction in patients with rheumatoid arthritis: a population-based cohort study. Ann Rheum Dis 2011;70:1020-4.

4. Provan SA, Semb AG, Hisdal J, et al. Remission is the goal for cardiovascular risk management in patients with rheumatoid arthritis: a cross-sectional comparative study. Ann Rheum Dis 2011;70:812-17.

5. Avina-Zubieta JA, Thomas J, Sadatsafavi M, et al. Risk of incident cardiovascular events in patients with rheumatoid arthritis: a meta-analysis of observational studies. Ann Rheum Dis 2012;70:1524-9.

6. Van Halm VP, Peters MJ, Voskuyl AE, et al. Rheumatoid arthritis versus diabetes as a risk factor for cardiovascular disease: a cross-sectional study, the CARRÉ Investigation. Ann Rheum Dis 2009;68:1395-400.

7. Solomon DH, Goodson NJ, Katz JN, et al. Patterns of cardiovascular risk in rheumatoid arthritis. Ann Rheum Dis 2006;65: 1608-12.

8. Holmqvist ME, Wedren S, Jacobsson LT, et al. Rapid increase in myocardial infarction risk following diagnosis of rheumatoid arthritis amongst patients diagnosed between 1995 and 2006. J Intern Med 2010;268:578-85.

9. Franklin J, Farragher TM, Lund M, et al. Excess risk of hospital admission for cardiovascular disease within the first 7 years from onset of inflammatory polyarthritis. Ann Rheum Dis 2010;69: $1660-4$.

10. Van Doornum S, Brand C, King B, et al. Increased case fatality rates following a first acute cardiovascular event in patients with rheumatoid arthritis. Arthritis Rheum.2006;54:2061-8.

11. Södergren A, Stegmayr B, Lundberg V, et al. Increased incidence of and impaired prognosis after acute myocardial infarction among patients with seropositive rheumatoid arthritis. Ann Rheum Dis 2007;66:263-6.

12. Fischer LM, Schlienger RG, Matter C, et al. Effect of rheumatoid arthritis or systemic lupus erythematosus on the risk of first-time acute myocardial infarction. Am J Cardiol 2004;93:198-200.

13. Ellingsen $\mathrm{T}$, Horslev-Petersen $\mathrm{K}$, Hetland ML, et al. Dyslipidaemia in early rheumatoid arthritis patients is common and not influenced by two years of effective DMARD therapy. The Opera Study. Arthritis Rheum 2012;64:S181.

14. Areskoug-Josefsson K, Oberg U. A literature review of the sexual health of women with rheumatoid arthritis. Musculoskeletal Care 2009;7:219-26.

15. Josefsson KA. Sexual health in patients with rheumatoid arthritis experiences, needs and communication with health care professionals. Musculoskeletal Care 2012;10:76-89.

16. Lee RK, Chughtai B, Te AE. Sexual function in men with metabolic syndrome. Urol Clin North Am 2012;39:53-62.

17. Smith BE, O'Connell DP, Patel DL, et al. Risk factors for erectile dysfunction in a cohort of 108477 Australian men. Med J Aust 2013;199:107-11.

18. Briganti VC, Jackson A, Kloner G, et al. A systematic review of the association between erectile dysfunction and cardiovascular disease. Eur Urol 2014;65:968-78. 
19. Shin D, Pregrenzer G, Gardin JM. Erectile dysfunction a disease marker for cardiovascular disease. Cardiol Rev 2011;19:5-11.

20. Gaede P, Lund-Andersen $\mathrm{H}, \mathrm{Parving} \mathrm{HH}$. Effect of a multifactorial intervention on mortality in type 2 diabetes. $N$ Engl J Med 2008;358:580-91.

21. Gaede $P$, Vedel $P$, Larsen $N$, et al. Multifactorial intervention and cardiovascular disease in patients with type 2 diabetes. $N$ Engl $J$ Med 2003;348:383-93.

22. Hansson L, Hedner T, Dahlof B. Prospective randomized open blinded end-point PROBE study. A novel design for intervention trials. Prospective Randomized Open Blinded End-Point. Blood Press 1992;1:113-19.

23. Reumatoidartrit-kliniske retningslinje; Dansk Reumatologisk Selskab. 2012. http://www.danskreumatologiskselskab.dk/date (accessed 8 June 2013).

24. Bang L, Bruun NE. Hypertensio arterialis- behandlingsvejledning. Dansk Hypertensions Selskab, 2009. http://www.dahs.dk/date (accessed 8 Jun 2013).

25. Snorgaard O, Drivsholm TB, Breum L, et al. Farmakologisk behandling af type 2-diabetes mål og algoritmer. Dansk Endokrinologisk Selskab. http://www.endocrinology.dk/date (accessed 8 Jun 2013)

26. Dansk Cardiologisk selskab. Forebyggelse af hjertesygdom. http:// www.cardio.dkdate (accessed 8 Jun 2013).

27. Aletaha D, Neogi T, Silman AJ, et al. 2010 Rheumatoid arthritis classification criteria: an American College of Rheumatology/ European League Against Rheumatism collaborative initiative. Arthritis Rheum 2010;62:2569-81.

28. Farnier M, Guyton JR, Jensen E, et al. Effects of ezetimibe, simvastatin and ezetimibe/simvastatin on correlations between apolipoprotein B, LDL cholesterol and non-HDL cholesterol in patients with primary hypercholesterolemia. Atherosclerosis 2013;229:415-42.

29. Clausen $P$, Jensen JS, Jensen $G$, et al. Elevated urinary albumin excretion is associated with impaired arterial dilatory capacity in clinically healthy subjects. Circulation 2001;103:1869-74.

30. Yudkin JS, Forrest JD, Jackson CA. Microalbuminuria as predictor of vascular disease in non-diabetic subjects. Islington Diabetes Survey. Lancet 1988;2:530-3.

31. Graham I, Atar D, Borch-Johnsen K, et al. European guidelines on cardiovascular disease prevention in clinical practice: executive summary: fourth Joint Task Force of the European Society of Cardiology and Other Societies on Cardiovascular Disease Prevention in Clinical Practice (Constituted by representatives of nine societies and by invited experts). Eur Heart $J$ 2007;28:2375-14.

32. Heartscore. http://www.heartscore.org/Date (access 8 June 2013).

33. Alkohol-Sundhedsstyrelsen. sundhedsstyrelsen.dk/da/sundhed/ alkoholdate (accessed 8 Jun 2013).

34. Hetland ML, Stengaard-Pedersen K, Junker P, et al. Combination treatment with methotrexate, cyclosporine, and intraarticular betamethasone compared with methotrexate and intraarticular betamethasone in early active rheumatoid arthritis: an investigatorinitiated, multicenter, randomized, double-blind, parallel-group, placebo-controlled study. Arthritis Rheum 2006;54:1401-9.

35. Avina Zubieta JA, Choi HK, Sadatsfavi M, et al. Risk of cardiovascular mortality in patients with rheumatoid arthritis: a meta-analysis of observational studies. Artritis Rheum 2008;59:1690-7.

36. Barnabe C, Martin BJ, Ghali WA. Systematic review and meta-analysis: anti-tumor necrosis factor? Therapy and cardiovascular events in rheumatoid arthritis. Arthritis Care Res (Hoboken) 2011;63:522-9.

37. Meune C, Touzé E, Trinquart L. High risk of clinical cardiovacular events in rheumatoid arthritis: levels of associations of myocardial infarction and stroke through a systematic meta-analysis. Arch Cardiovasc Dis 2010;103:253-61.

38. Keller A, McGarvey EL, Clayton AH. Reliability and construct validity of the changes in sexual functioning questionnaire short-form (CSFQ-14). J Sex Marital Ther 2006;32:43-52.

39. Christensen BS, Grønbaek M, Osler M, et al. Sexual dysfunctions and difficulties in Denmark: prevalence and associated sociodemographic factors. Arch Sex Behav 2011;40:121-32.

40. Bech P, Rasmussen NA, Olsen LR, et al. The sensitivity and specificity of the major depression inventory, using the present state examination as the index of diagnostic validity. $J$ Affect Disord 2001;66:159-64.

41. Schefte DB, Hetland ML. An open-source, self-explanatory touch screen in routine care. Validity of filling in the Bath measures on Ankylosing Spondylitis Disease Activity Index, Function Index, the Health Assessment Questionnaire and Visual Analogue Scales in comparison with paper versions. Rheumatology (Oxford) 2010;49:99-104.

42. Hørslev-Petersen $\mathrm{K}$, Hetland ML, Junker $\mathrm{P}$, et al. Adalimumab added to a treat-to-target strategy with methotrexate and intra-articular triamcinolone in early rheumatoid arthritis increased remission rates, function and quality of life. The OPERA Study: an investigator-initiated, randomised, double-blind, parallel-group, placebo-controlled trial. Ann Rheum Dis 2014;73:654-61.

43. Linde L, Sørensen J, Østergaard M, et al. What factors influence the health status of patients with rheumatoid arthritis measured by the SF-12v2 Health Survey and the Health Assessment Questionnaire? $J$ Rheumatol 2009;36:2183-9.

44. Semb AG, Kvien TK, DeMicco DA, et al. Effect of intensive lipid-lowering therapy on cardiovascular outcome in patients with and those without inflammatory joint disease. Arthritis Rheum.2012;64:2836-46.

45. Mancia G, Fagard R, Narkiewicz K, et al, Task Force Members. 2013 Practice guidelines for the management of arterial hypertension of the European Society of Hypertensgon (ESH) and the European Society of Cardiology (ESC): ESH/ESC Task Force for the Management of Arterial Hypertension. $J$ Hypertens 2013;31:1925-38

46. Kurth T, de Jong PE, Cook NR, et al. Kidney function and risk of cardiovascular disease and mortality in women: a prospective cohort study. BMJ 2009;338:b2392.

47. Helsinki Declaration. http://www.wma.net/en/30publications/ 10policies/b3date (accessed 8 Jun 2013).

48. Lv S, Liu Y, Zou Z, et al. The impact of statins therapy on disease activity and inflammatory factor in patients with rheumatoid arthritis: a meta-analysis. Clin Exp Rheumatol 2015;33:69-76.

49. van Sijl AM, van den Oever IA, Peters MJ, et al. Subclinical renal dysfunction is independently associated with cardiovascular events in rheumatoid arthritis: the CARRÉ Study. Ann Rheum Dis 2012:71:341-4.

50. Gaede $\mathrm{P}$, Vedel $\mathrm{P}$, Parving $\mathrm{HH}$, et al. Intensified multifactorial intervention in patients with type 2 diabetes mellitus and microalbuminuria: the Steno type 2 randomised study. Lancet 1999;353:617-22

51. Arts EE, Popa C, Den Broeder AA, et al. Performance of four current risk algorithms in predicting cardiovascular events in patients with early rheumatoid arthritis. Ann Rheum Dis 2015;74:668-74.

52. Mäki-Petäjä KM, Booth $A D$, Hall FC, et al. Ezetimibe and simvastatin reduce inflammation, disease activity, and aortic stiffness and improve endothelial function in rheumatoid arthritis. J Am Coll Cardiol 2007:50:852-8.

53. Kitas GD, Nightingale P, Armitage J, et al. Trial of Atorvastatin for the primary prevention of cardiovascular events in patients with $R A$ (TRACE RA): a randomized controlled trial in 2986 RA patients. Rheumatology 2015;54(Suppl 1):i87. 